\title{
Unequal Neighbourhoods, Unequal Schools? Organisational Neighbourhood Inequality and the Question of Organisational Habitus and Organisational Practices. In Unequal Neighbourhoods, Unequal Schools
}

\author{
Autores: Julia Nast \\ Editorial: Springer VS \\ Año de publicación: 2020 \\ $N^{o}$ de páginas: 285 \\ ISBN: 978-3-658-27591-4
}

Julia Nast es la autora del libro Unequal Neighbourhoods, Unequal Schools? Organisational Neighbourhood Inequality and the Question of Organisational Habitus and Organisational Practices. In Unequal Neighbourhoods, Unequal Schools (¿Barrios desiguales, escuelas desiguales? Desigualdad de barrios y la cuestión del hábito organizativo y las prácticas organizativas. En barrios Desiguales, Escuelas Desiguales en español). Un libro de 285 páginas que tiene como base los resultados principales de su tesis. La autora es doctora en Sociología por la Universidad de Humbold (Berlín) y el King's College London (Inglaterra). Su área de especialidad es la educación, la desigualdad y el papel de los barrios en estos campos.

En esta obra, el lector o la lectora puede adentrarse en la exploración de diferentes barrios a través de una aproximación etnográfica, que se completa con entrevistas, realizada en dos centros educativas de primaria de la ciudad de Berlín (Alemania). Los resultados demuestran, finalmente, cómo la práctica docente cambia según el contexto y lo que esto representa para el barrio y la desigualdad.

Este volumen está dividido en los siguientes siete capítulos (todos traducidos al español) que se presentan en el mismo orden que aparecen en la obra, todos ellos divididos en diferentes subsecciones que permite al lector profundizar en los mismos:

El primer capítulo, titulado ¿Vecindarios desiguales, escuelas desiguales? Desigualdad de vecindario y la cuestión de la organización. Hábito y prácticas organizativas, es una introducción que permite un acercamiento previo al trabajo de investigación realizado por Nast. En él pueden leerse diferentes apartados que resumen el tema de desigualdad organizativa de los barrios, las prácticas organizativas de las organizaciones del barrio. El capítulo introductorio que, además de una aproximación teórica, ofrece un pequeño resumen del enfoque metodológico.

El segundo capítulo, Barrios, escuelas y desigualdad: Cambiando el enfoque, es utilizado por la autora a modo de marco teórico, en el que permite que el lector conozca sobre la desigualdad urbana, los límites que tienen las organizaciones y los efectos del barrio en las mismas. También da las claves necesarias para conocer las organizaciones a partir de la investigación urbana, clave si el lector no es del campo de la sociología, pues permite comprender cuáles son las cantidades de organizaciones y la calidad de las mismas de aquellos barrios de mayor pobreza. Nast cierra el capítulo adentrándose en las escuelas y dedica quince páginas para dar a conocer la relación entre los barrios, los centros educativos y la desigualdad.

El capítulo tercero, Una perspectiva teórica: Campos localizados, organización. Hábito y prácticas organizativas, está dedicado a conceptualizar la relación entre las organizaciones y los barrios. Este capítulo tiene un corte sociológico que permite conocer más sobre las organizaciones: sus estructuras, su organización, las prácticas que llevan a cabo, que permite comprender cómo es la desigualdad organizativa de los barrios

El cuarto capítulo presenta el trabajo metodológico: los datos, la metodología etnográfica y el trabajo de campo. Este trabajo de campo se ha realizado en dos barrios concretos: Cross-Square y Roseville. Se explica también cuáles fueron las razones para elegir estos barrios y se hace una comparación entre las escuelas de estos vecindarios. Al final del libro se encuentra como anexo un índice con las preguntas de las entrevistas.

El quinto capítulo, Cómo los barrios dan forma a las escuelas como campos: Social, Diferencias simbólicas $y$ administrativas. Este capítulo se divide en cuatro secciones. La primera trata sobre el poder y las unidades sociales (familias y docentes) y sobre las presiones de carácter institucional, la desigualdad y la meritocracia. Una segunda sección está enfocada a la presión institucional de los barrios estudiados. La tercera parte del capítulo se centra en los proyectos, cooperación y la integración institucional de los dos barrios estudiados. Y una última parte en la que ayuda a que el lector o lectora comprenda cómo la escuela centra parte de la desigualdad del barrio. 
El capítulo penúltimo, Cómo se adaptan los profesionales de la educación: Organización localizada. Hábito y prácticas organizativas. Este tiene una mirada muy centrada en las prácticas que se llevan a cabo desde las organizaciones. Por un lado, se centra en las prácticas de aspecto emocional y la promoción de autonomía entre los estudiantes. La autora refleja en estas páginas cómo la desigualdad está aceptada y los profesionales de la escuela trabajan con un enfoque en el que han asumido que no pueden "salvar" a todos sus estudiantes. Asimismo, resalta cómo influye los padres en la organización escolar de los docentes y cómo estos hacen mejoras en sus prácticas educativas. También durante este capítulo se invita a que el lector o lectora reflexione sobre los problemas de desigualdad: ¿son casos aislados o es que en realidad son problemas sociales que no han recibido respuesta?

El último capítulo se titula Desigualdad organizativa de los barrios y qué hacer Acerca de eso. Para cerrar la obra, Julia Nast propone un enfoque nuevo que nace de una perspectiva teórica y a través de la reflexión del impacto de los barrios en las prácticas que se dan en las organizaciones. También hace una reflexión crítica sobre su propio trabajo, y en esta sección Nast argumenta cuáles han sido las limitaciones que ha tenido para realizar esta investigación.

En definitiva, esta obra nos aporta una visión sociológica de gran interés sobre los barrios vulnerables y las instituciones que se encuentran en ellos. La segregación aumenta en muchos núcleos urbanos y la autora propone una agenda de investigación para la desigualdad organizativa de los barrios. La intención de Nast es clara: dar a conocer cómo la praxis de los docentes cambia según el contexto en el que se encuentran trabajando y cómo esto repercute reproduciendo, más aún, la desigualdad urbana.

Irene Moreno-Medina

Universidad Autónoma de Madrid irene.morenom@uam.es 